\title{
Failure Probability Modeling of Miniature DC Motors and Its Application in Fault Diagnosis
}

\author{
Zhiping Xie $\mathbb{D}^{D}$, Rongchen Zhao $\mathbb{D}$, Jiming Zheng, and Yancheng Lang \\ School of Mechanical \& Electrical Engineering, Guizhou Normal University, Guiyang 550001, China \\ Correspondence should be addressed to Rongchen Zhao; zhaorongchen99@gmail.com
}

Received 28 March 2021; Accepted 18 May 2021; Published 8 October 2021

Academic Editor: Mickael Lallart

Copyright (c) 2021 Zhiping Xie et al. This is an open access article distributed under the Creative Commons Attribution License, which permits unrestricted use, distribution, and reproduction in any medium, provided the original work is properly cited.

\begin{abstract}
This paper proposes a fault diagnosis method for miniature DC motors (MDCMs) in the presence of the uncertainties caused by material and random factors of the production process. In this method, the probability models of fault multiple features are established based on the advantage criterion of the maximum overall average membership to determine the distribution of fault multiple features. The fault diagnosis algorithm is synthesized to obtain the threshold ranges of fault multiple features according to different confidence levels. Experimental test results are presented and analyzed to validate the efficiency and performance of the proposed fault diagnosis method.
\end{abstract}

\section{Introduction}

Due to their small size, lightweight, and easy control, miniature DC motors (MDCMs) are widely used in the industrial fields of home appliances, office automation, automotive parts, etc. [1]. In these applications, the efficient and reliable operation of MDCMs is increasingly important. However, the factory quality inspection of MDCMs mainly relies on the manual experience, which leads to low production efficiency, heavy workload, and missed inspection [2]. Therefore, the effective motor fault detection technology on the production line is crucial for the factory quality of MDCMs.

Generally, there are four main techniques for the motor fault diagnosis, which are classified into the signal-based, model-based, data-based, and indicator-based method classes [3, 4]. The signal-based method uses sensor equipment to measure the diagnostic signals of the motor, such as current [5], vibration [6], acoustic [7], and thermal [8], and the fault type is detected by comparing and analyzing the diagnostic signals. Measurements of the acoustic signal, vibration signal, and thermal signal are noninvasive. The motor current signature analysis (MCSA) has been successfully applied to the fault diagnosis of DC motors and induction motors, especially under steady-state conditions and rated load [9]. However, the signal-based method requires a large number of sensing devices.

The model-based method relies on the theoretical analysis of the motor, which key is to establish the motor model under the fault condition [3]. The motor fault can be detected by comparing the actual parameters with the estimated parameters of the properly functioning motor. Residual analysis and suitable signal processing are used to define the fault indicators [3]. Winding function approach [10], dynamic mesh reluctance approach [3], and finite element approach [11] were used to model. The available models are nice analytical tools predicting motor behavior, but their parameters are not easily determined. The leastsquares (LS) method [11, 12], fully decoupled parity equation [13], and universal adaptive stabilizer [14] were used to estimate the motor parameters. Since the motor faults generally change multiple electromechanical parameters, it is difficult to establish a fault prediction model of the complex fault systems.

The data-based method is a powerful tool to improve the effectiveness and reliability of the diagnosis since it does not require any knowledge about motor parameters and models $[3,4]$. Artificial intelligence techniques are widely used in the 
data-based method, which need to use the healthy and faulty motor data for feature extraction and classification. Several artificial intelligence techniques, such as neural networks $[15,16]$, support vector machine [15], fuzzy logic [17], expert system networks [18], deep learning [19], and algorithms [20], have been developed to detect the motor faults. The combinations of the above techniques have been reported in the literature. The convolutional neural network (CNN) was used for extracting features, and then the support vector machine [21] and deep transfer learning [22] were used to diagnose faults. The application of artificial intelligence techniques in the motor fault diagnosis can promote the automation of the diagnostic process. However, these methods require a large set of training samples [23], and the generalization performance of artificial intelligence techniques has limitations.

The indicator-based method is to compare the measured value with the indicator threshold, which is a widely used fault diagnosis method. An and Li [24] proposed the permutation entropy of the reconstructed signal as the fault feature of piezoelectric ceramics. Jafari et al. [25] established four different indices and used the well-established Otsu thresholding technique to set the index thresholds for fault diagnosis. Ali and Liang [26] used d8 for the fault indicator, and the indicator threshold was determined using the universal threshold technique. Irfan et al. [27] designed an adaptive threshold scheme using the statistical decision theory. An accurate indicator threshold is essential for the indicator-based method. In addition, in the manufacturing process of MDCMs, various uncontrollable factors (material nonlinearity and random factors of the production process) lead to uncertainties of the MDCM output performance. Therefore, a fixed indicator threshold is difficult to apply to the fault diagnosis of the MDCM.

Fault diagnosis methods of the MDCM need to consider the uncertainty of the production process. Wang et al. [23] used a probability modeling approach for photovoltaic fault diagnosis to solve the problems of the nonlinearity and uncertainty of the PV array output interval. Liu et al. [28] proposed a failure probability calculation method for power equipment based on multicharacteristic parameters. Li et al. [29] proposed an adaptive dynamic update model of the equipment alarm threshold based on a similar proportion and state probability model. Wen and Gao [30] estimated the probability distribution of health indicator points, and the deterioration of the ball screw is evaluated by the probability distribution. The probability density function of fault indicators can be determined using the error comparison method [23] and kernel density estimation $[29,30]$.

However, the probabilistic fault diagnoses of DC motors have not been considered in the previous studies. This paper proposes a novel solution to apply a fault diagnosis method for the MDCM by incorporating the probability modeling and the advantage criterion of the maximum overall average membership.

The novelties and contributions of this paper are summarized as follows:
(1) Proposing the advantage criterion of the maximum overall average membership to determine the optimal fitting distribution, which cannot be handled by the traditional hypothesis test

(2) Designing a fault diagnosis algorithm based on probability modeling to deal with the uncertainties caused by material and random factors of the production process, improving the accuracy of MDCM fault diagnosis

(3) Designing the diagnostic test platform of MDCMs to validate the performance of the proposed fault diagnosis method

The rest of this paper is arranged as follows: in Section 2, the structure of the MDCM and data sources are described in detail. In Section 3, the probability models of fault multiple features are established using the advantage criterion of the maximum overall average membership, and the fault diagnosis method is discussed in detail, which is verified in Section 4. Section 5 summarizes this paper and outlines future research directions.

\section{Data Acquisition}

2.1. Structure of the MDCM. Figure 1 shows the schematic diagram of the MDCM discussed in this paper. The MDCM consists of a rotor and a stator (Figure 1(a)). The stator is equipped with a ring permanent magnet, and the rotor consists of three coil windings which are wounded on three teeth [31]. The motor windings are connected by a triangle connection (Figure 1(b)). The parameters of a healthy MDCM used for testing are listed in Table 1.

2.2. Data Sources. By analyzing and solving the mathematical model of the MDCM, the fault multiple features, namely, DC component $\left(P_{d c}\right)$, frequency point $\left(f_{m}\right)$, amplitude of frequency point $\left(P_{f}\right)$, and spectrum area $(A)$, were proposed to diagnose motor faults in [2]. Figure 2 shows the structure diagram of data acquisition of the MDCM with no load. After preamplification, low-pass filtering, analog-todigital (A/D) converter, and fast Fourier transform (FFT) conversion, the frequency domain data of fault multiple features $\left(P_{d c}, f_{m}, P_{f}\right.$, and $\left.A\right)$ can be obtained by the data statistics [2]. In this paper, the healthy motor (HM), the interturn short fault motor (ISFM), the open winding fault motor (OWFM), the winding desoldering fault motor (WDFM), and the abnormal magnet loop fault motor (AMFM) were studied. 110 healthy motors and 60 motors (Xiamen Dazhen Electric Co., Ltd.) with each fault type were randomly selected as experimental samples.

\section{Fault Diagnosis Method}

3.1. Maximum Overall Average Membership Advantage Criterion. The advantage criterion of the maximum overall average membership was used to determine the distribution of the fault multiple features [32, 33]. The key to the 


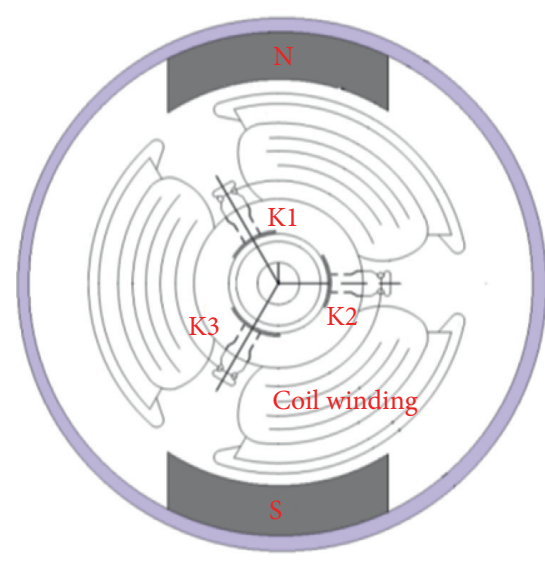

(a)

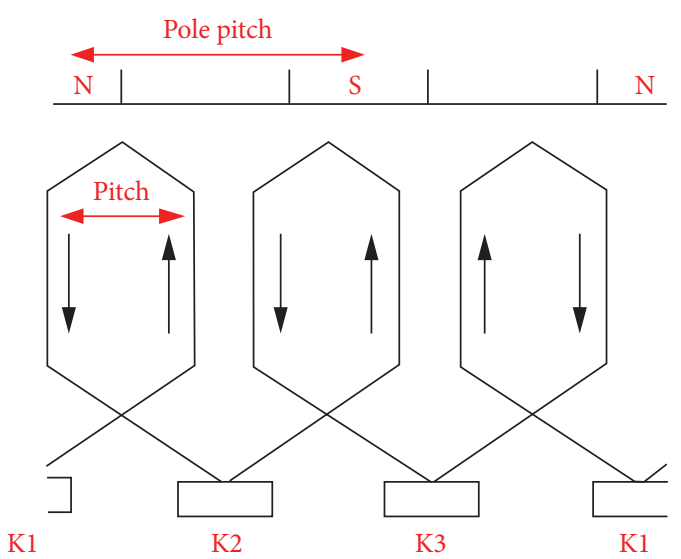

(b)

Figure 1: Schematic diagram of the MDCM: (a) structure of the MDCM; (b) coil winding diagram.

TABLE 1: Main parameters of a healthy MDCM.

\begin{tabular}{lc}
\hline Motor parameters & Values \\
\hline Rated voltage & $5.0 \mathrm{~V} \mathrm{DC}$ \\
No load current & $45 \mathrm{~mA} \mathrm{max}$ \\
No load speed & $5400-6700 \mathrm{r} / \mathrm{min}$ \\
Phase resistance of the winding & $18 \Omega$ \\
Moment of inertia & $2.668 \times 10-7 \mathrm{kgm}^{2}$ \\
Damping coefficient & $4 \times 10^{-7}$ \\
Number of poles & 2 \\
\hline
\end{tabular}

advantage criterion of the maximum overall average membership is to construct membership functions.

Step 1. Determine the membership function.

Let the domain of the fault multiple features' distribution model be $U$, and $\widetilde{A}_{1}, \widetilde{A}_{2}, \widetilde{A}_{3}$, and $\widetilde{A}_{4}$ denote the normal distribution, exponential distribution, Weibull distribution, and lognormal distribution, respectively. They are the fuzzy subset of $F(U) . Y$ is a sample of $U$, $Y=\left\{y_{1}, y_{2}, \ldots, y_{n}\right\}^{T}$, where $n$ is the number of motor samples. The membership function $\mu_{\tilde{A}_{i}}\left(y_{j}\right) \quad(i=1,2,3,4)$ represents the fuzzy membership degree of the $y_{j}$ th index in the sample $Y$ to the fuzzy subset $\widetilde{A}_{i}$. The normalization of $\mu_{\hat{A}_{i}}\left(y_{j}\right)$ is as follows:

$$
\begin{array}{r}
\mu_{\widetilde{A}_{1}}\left(y_{j}\right)+\mu_{\tilde{A}_{2}}\left(y_{j}\right)+\mu_{\tilde{A}_{3}}\left(y_{j}\right)+\mu_{\widetilde{A}_{4}}\left(y_{j}\right)=1, \\
j=1,2, \ldots, n .
\end{array}
$$

Normal distribution membership function $\mu_{\tilde{A}_{1}}\left(y_{j}\right)$ is expressed as

$$
\mu_{\tilde{A}_{1}}\left(y_{j}\right)=\frac{F_{1}\left(y_{j}\right)}{F_{1}\left(y_{j}\right)+F_{2}\left(y_{j}\right)+F_{3}\left(y_{j}\right)+F_{4}\left(y_{j}\right)},
$$

where

$$
\begin{aligned}
F_{1}\left(y_{j}\right) & =1-\left|\varnothing\left(\frac{y_{j}-\mu}{\sigma}\right)-F\left(y_{j}\right)\right|, \\
\varnothing\left(\frac{y_{j}-\mu}{\sigma}\right) & =\int_{-\infty}^{y_{j}-\mu / \sigma} \frac{1}{\sqrt{2 \pi}} e^{-t^{2} / 2} \mathrm{~d} t, \\
F_{2}\left(y_{j}\right) & =1-\left|1-e^{-y_{j} / \lambda}-F\left(y_{j}\right)\right|, \\
F_{3}\left(y_{j}\right) & =1-\left|1-e^{-\left(y_{j} / \eta\right)^{\beta}}-F\left(y_{j}\right)\right|, \\
F_{4}\left(y_{j}\right) & =1-\left|\varnothing\left(\frac{\ln y_{j}-\mu_{l}}{\sigma_{l}}\right)-F\left(y_{j}\right)\right|, \\
\varnothing\left(\frac{\ln y_{j}-\mu_{l}}{\sigma_{l}}\right) & =\int_{-\infty}^{\mathrm{n} y_{j}-\mu_{l} / \sigma_{l}} \frac{1}{\sqrt{2 \pi}} e^{-t^{2} / 2} \mathrm{~d} t,
\end{aligned}
$$

where $\varnothing\left(y_{j}-\mu / \sigma\right)$ and $F\left(y_{j}\right)$ are the distribution function of the normal distribution and the empirical cumulative distribution function of the sample data, respectively. Parameters $\sigma$ and $\mu$ are estimated from the sample data. $1-e^{-y_{j} / \lambda}$ is the distribution function of the exponential distribution, and the parameter $\lambda$ is estimated from the sample data. $1-e^{-\left(y_{j} / \eta\right)^{m}}$ is the distribution function of the Weibull distribution, and the parameters $\eta$ and $m$ are estimated from the sample data. $\varnothing\left(\ln y_{j}-\mu / \sigma\right)$ is the distribution function of the lognormal distribution, and the parameters $\sigma_{l}$ and $\mu_{l}$ are estimated from the sample data.

Exponential distribution membership function $\mu_{\tilde{A}_{2}}\left(y_{j}\right)$ is expressed as

$$
\mu_{\tilde{A}_{2}}\left(y_{j}\right)=\frac{F_{2}\left(y_{j}\right)}{F_{1}\left(y_{j}\right)+F_{2}\left(y_{j}\right)+F_{3}\left(y_{j}\right)+F_{4}\left(y_{j}\right)} .
$$

Weibull distribution membership function $\mu_{\tilde{A}_{3}}\left(y_{j}\right)$ is expressed as

$$
\mu_{\tilde{A}_{3}}\left(y_{j}\right)=\frac{F_{3}\left(y_{j}\right)}{F_{1}\left(y_{j}\right)+F_{2}\left(y_{j}\right)+F_{3}\left(y_{j}\right)+F_{4}\left(y_{j}\right)} .
$$



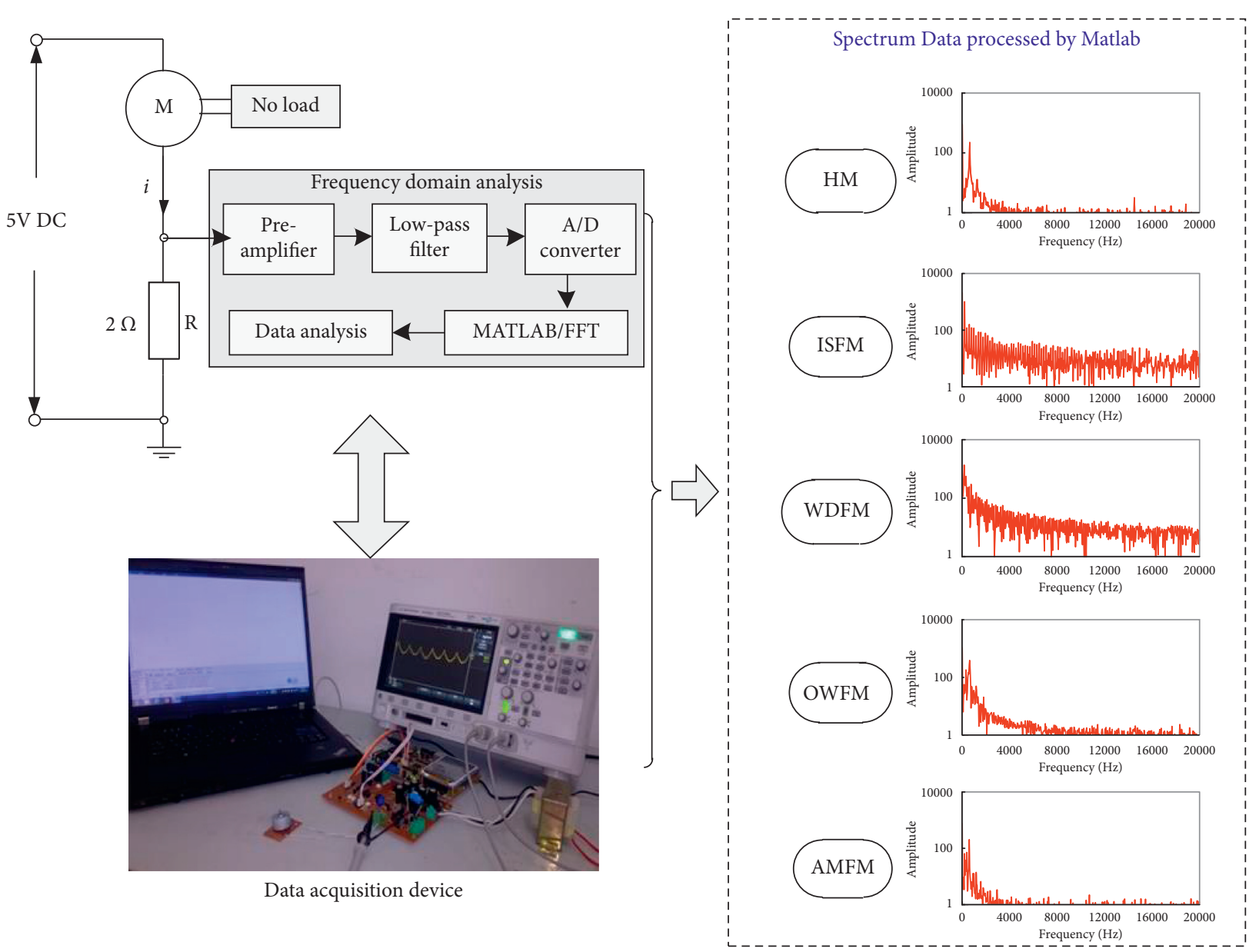

FIgure 2: Data acquisition of the MDCM.

Logarithmic normal distribution membership function $\mu_{A_{4}}\left(y_{j}\right)$ is expressed as

$$
\mu_{\widetilde{A}_{4}}\left(y_{j}\right)=\frac{F_{4}\left(y_{j}\right)}{F_{1}\left(y_{j}\right)+F_{2}\left(y_{j}\right)+F_{3}\left(y_{j}\right)+F_{4}\left(y_{j}\right)} .
$$

Step 2. Calculate the local membership advantage.

The local membership advantage $\Delta \mu_{\tilde{A}_{i}}\left(x_{j}\right)$ is defined as

$$
\begin{array}{r}
\Delta{\tilde{A_{i}}}_{i}\left(x_{j}\right)=\mu_{\tilde{A}_{k}}^{(i)}\left(y_{j}\right)-\min \left\{\mu_{\hat{A}_{i}}\left(y_{j}\right)\right\}, \\
i=1,2,3,4 ; j=1,2, \ldots, n,
\end{array}
$$

where $\mu_{\sim}^{(i)}\left(y_{j}\right)$ represents the new sequence of $\mu_{\tilde{A}}\left(y_{j}\right)$ sorted from the ${ }^{A_{k}}$ largest to the smallest. $k$ represents the sort of the new sequence, and $i$ represents the sort of the original sequence.

Step 3. Calculate the overall average membership advantage.

The overall average membership advantage $\mu_{\tilde{A}_{i}}(Y)$ is defined as

$$
\mu_{\tilde{A}_{i}}(Y)=\frac{1}{n} \sum_{j=1}^{n} \Delta \mu_{\tilde{A}_{i}}\left(y_{j}\right), \quad i=1,2,3,4 .
$$

Step 4. Calculate the maximum overall average membership advantage.

The maximum overall average membership advantage $\mu_{\tilde{A}}(Y)$ is defined as

$$
\mu \widetilde{A}(Y)=\max \left\{\mu_{\tilde{A}_{1}}(Y), \mu_{\tilde{A}_{2}}(Y), \mu_{\widetilde{A}_{3}}(Y), \mu_{\widetilde{A}_{4}}(Y)\right\} .
$$

3.2. Distribution Characteristics of Fault Multiple Features. Figure 3 shows the process of determining the distribution of fault multiple features. The maximum likelihood estimation method was used to estimate the parameters of the hypothetical distribution of fault multiple features. The advantage criterion of the maximum overall average membership was used to determine the distribution type of fault multiple features. The overall average membership advantage $\mu_{\tilde{A}_{i}}(Y)$ of fault multiple features was calculated based on the experimental data. The results of the hypothetical distribution of the fault multiple features are shown in Table 2. Because the resolution of the current spectrum is $40 \mathrm{~Hz}$ and the data interval of $f_{m}$ is narrow, $f_{m}$ does not meet any hypothetical distribution. Therefore, it is not necessary to confirm its distribution using the advantage criterion of the maximum overall average membership. 


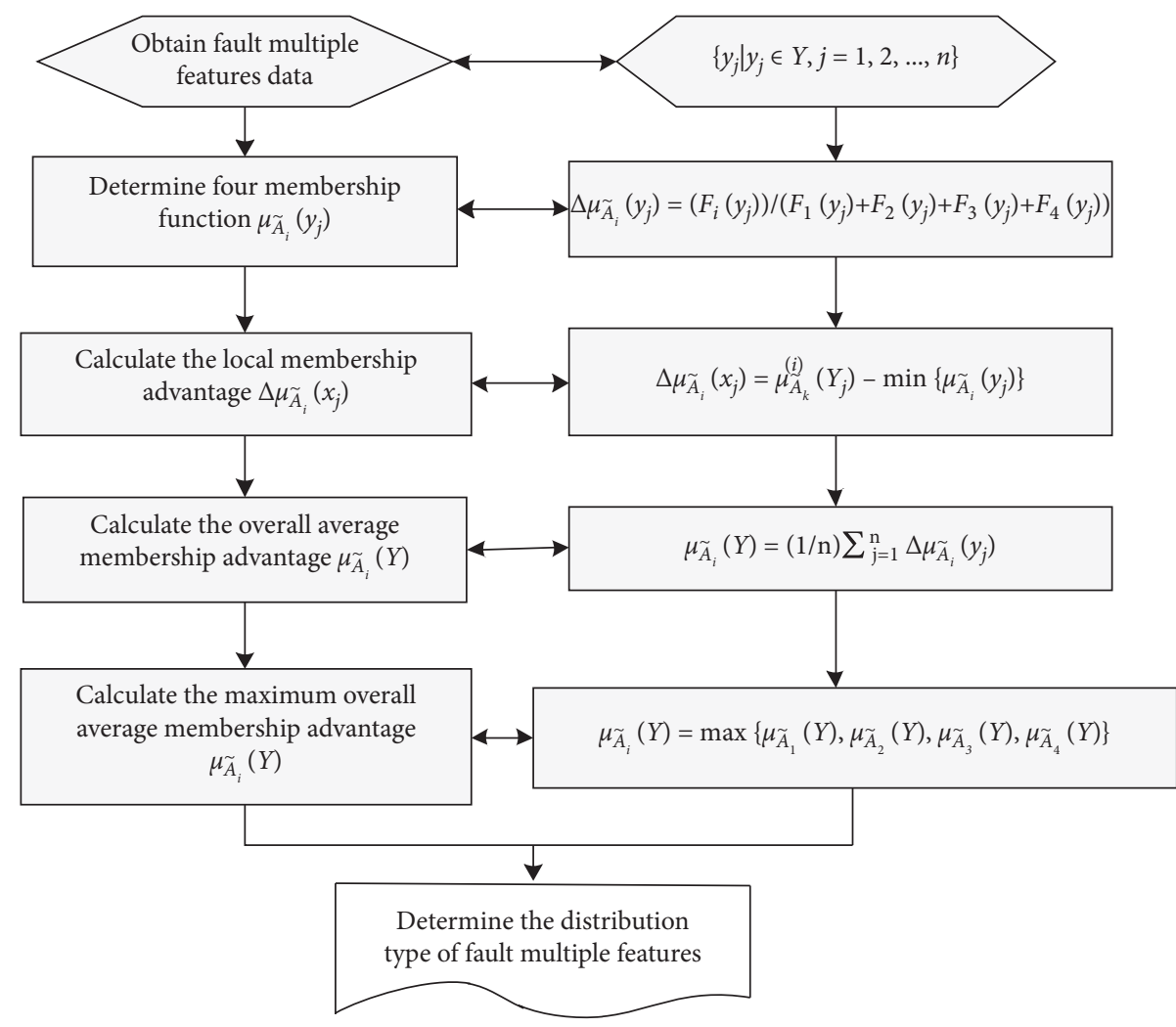

Figure 3: The flowchart of determining the distribution of fault multiple features.

TABle 2: Results of the hypothetical distribution.

\begin{tabular}{|c|c|c|c|c|c|c|c|c|c|}
\hline \multirow{3}{*}{ Type of fault } & \multirow{3}{*}{ Indicators } & \multicolumn{8}{|c|}{ Hypothetical distribution } \\
\hline & & \multicolumn{2}{|c|}{ Normal } & \multicolumn{2}{|c|}{ Exponential } & \multicolumn{2}{|c|}{ Weibull } & \multicolumn{2}{|c|}{ Lognormal } \\
\hline & & $\mu, \sigma$ & $\mu_{\tilde{A}_{1}}(Y)$ & $\lambda$ & $\mu_{\tilde{A}_{2}}(Y)$ & $\eta, \beta$ & $\mu_{\tilde{A}_{3}}(Y)$ & $\mu_{l}, \sigma_{l}$ & $\mu \tilde{A}_{4}(Y)$ \\
\hline \multirow{3}{*}{$H M$} & $P_{d c}$ & $893.82,59.98$ & 0.0667 & 893.82 & 0.0009 & $922.32,15.07$ & 0.0607 & $6.79,0.067$ & 0.0673 \\
\hline & $P_{\mathrm{f}}$ & $222.16,35.44$ & 0.0562 & 222.16 & 0.0010 & $237.08,7.27$ & 0.0580 & $5.39,0.166$ & 0.0531 \\
\hline & $A$ & $809.02,200.36$ & 0.0484 & 809.02 & 0.0011 & $887.39,4.38$ & 0.0472 & $6.67,0.250$ & 0.0487 \\
\hline \multirow{3}{*}{ ISFM } & $P_{d c}$ & $2103.8,84.30$ & 0.0674 & 2103.8 & 0.0026 & $2144.8,24.6$ & 0.0609 & $7.65,0.040$ & 0.0679 \\
\hline & $P_{\mathrm{f}}$ & $1050.22,54.76$ & 0.0669 & 1050.22 & 0.0005 & $1075.34,21.94$ & 0.0650 & $6.96,0.053$ & 0.065 \\
\hline & $A$ & $5632.8,148.47$ & 0.0709 & 5632.8 & 0.0011 & $688.19,11.09$ & 0.0643 & $8.64,0.026$ & 0.0622 \\
\hline \multirow{3}{*}{ OWFM } & $P_{d c}$ & $661.78,56.39$ & 0.0631 & 659.58 & 0.0003 & $679.73,16.19$ & 0.0546 & $6.49,0.084$ & 0.065 \\
\hline & $P_{\mathrm{f}}$ & $185.92,23.38$ & 0.0613 & 185.92 & 0.0003 & $196.12,8.98$ & 0.0589 & $5.22,0.129$ & 0.0599 \\
\hline & $A$ & $861.74,53.19$ & 0.0659 & 861.74 & 0.0017 & $887.19,15.60$ & 0.0580 & $6.76,0.061$ & 0.0647 \\
\hline \multirow{3}{*}{ WDFM } & $P_{d c}$ & $915.66,58.57$ & 0.0657 & 915.66 & 0.0021 & $943.87,14.56$ & 0.0562 & $6.82,0.063$ & 0.0654 \\
\hline & $P_{\mathrm{f}}$ & $1355.33,70.9$ & 0.0686 & 1355.33 & 0.0019 & $1389.57,18.92$ & 0.0618 & $7.21,0.052$ & 0.0696 \\
\hline & $A$ & $9780.93,228.4$ & 0.0701 & 9780.93 & 0.0022 & $9895.76,39.81$ & 0.0609 & $9.19,0.023$ & 0.0648 \\
\hline \multirow{3}{*}{$A M F M$} & $P_{d c}$ & $1072.4,97.79$ & 0.0627 & 1072.4 & 0.0005 & $1117,11.8$ & 0.0607 & $6.97,0.092$ & 0.0621 \\
\hline & $P_{\mathrm{f}}$ & $380.54,31.72$ & 0.0629 & 380.54 & 0.0004 & $394.56,14.60$ & 0.0638 & $5.94,0.085$ & 0.0622 \\
\hline & $A$ & $2662.9,139.8$ & 0.0667 & 2662.9 & 0.0025 & $2730.83,18.72$ & 0.0604 & $7.89,0.052$ & 0.063 \\
\hline
\end{tabular}

It can be seen from Table 2 that $\mu_{\tilde{A}_{4}}(Y)$ of $P_{d c}$ is the largest among all of them for HM, and it obeys lognormal distribution according to the advantage criterion of the maximum overall average membership. $P_{f}$ and $A$ obey the Weibull distribution and lognormal distribution, respectively. For ISFM, OWFM, WDFM, and AMFM, the process of determining the distribution of fault multiple features is similar to that of HM.
3.3. Probability Density Function of Fault Multiple Features. The probability density functions of the fault multiple features for HM, ISFM, OWFM, WDFM, and AMFM are shown in Table 3. It can be seen from Table 3 that the fault multiple features obey normal distribution, lognormal distribution, and Weibull distribution, respectively.

The probability density function diagrams of the fault multiple features in different conditions are shown in 
TABle 3: Probability density function of the fault multiple features.

\begin{tabular}{lccc}
\hline Type of fault & \multicolumn{1}{c}{$P_{d c}$} & $P_{\mathrm{f}}$ & $A$ \\
\hline HM & $1 / \sqrt{2 \pi} \times 0.067 x e^{-(\ln x-6.79)^{2} / 2 \times 0.067^{2}}$ & $7.27 / 237.08(x / 237.08)^{6.27} e^{-(x / 237.08)^{7.27}}$ & $1 / \sqrt{2 \pi} \times 0.025 x e^{-(\ln x-6.67)^{2} / 2 \times 0.025^{2}}$ \\
ISFM & $1 / \sqrt{2 \pi} \times 0.04 x e^{-(\ln x-7.65)^{2} / 2 \times 0.04^{2}}$ & $1 / \sqrt{2 \pi} \times 54.76 e^{-(x-1050.22)^{2} / 2 \times 54.76^{2}}$ & $1 / \sqrt{2 \pi} \times 148.47 e^{-(x-5632.8)^{2} / 2 \times 148.47^{2}}$ \\
OWFM & $1 / \sqrt{2 \pi} \times 0.084 x e^{-(\ln x-6.49)^{2} / 2 \times 0.084^{2}}$ & $1 / \sqrt{2 \pi} \times 23.38 e^{-(x-185.92)^{2} / 2 \times 23.38^{2}}$ & $1 / \sqrt{2 \pi} \times 53.19 e^{-(x-861.74)^{2} / 2 \times 53.19^{2}}$ \\
WDFM & $1 / \sqrt{2 \pi} \times 58.57 e^{-(x-915.66)^{2} / 2 \times 58.57^{2}}$ & $1 / \sqrt{2 \pi} \times 0.052 x e^{-(\ln x-7.21)^{2} / 2 \times 0.052^{2}}$ & $1 / \sqrt{2 \pi} \times 0228.4 e^{-(x-9780.93)^{2} / 2 \times 0228.44^{2}}$ \\
AMFM & $1 / \sqrt{2 \pi} \times 97.79 e^{-(x-1072.4)^{2} / 2 \times 97.79^{2}}$ & $14.6 / 394.56(x / 394.56)^{13.6} e^{-(x / 394.56)^{14.6}}$ & $1 / \sqrt{2 \pi} \times 139.8 e^{-(x-2662.9)^{2} / 2 \times 139.8^{2}}$ \\
\hline
\end{tabular}

Figure 4. The characteristics of the probability density function are summarized as follows:

(1) The distribution of each fault multiple feature changes with the type of fault.

(2) There is no obvious regularity in the distribution of fault multiple features.

(3) It can clearly distinguish HM, ISFM, and WDFM using fault multiple features.

(4) The probability density curves of HM and OWFM have overlapping areas. Through the setting threshold range of $P_{d c}$, it is possible to distinguish between HM and OWFM.

3.4. Threshold of Fault Multiple Features. The probability distribution of fault multiple features with different fault types was determined using the probability statistics and the advantage criterion of the maximum overall average membership. The threshold range of fault multiple features with different fault types can be calculated by the following equation:

$$
P\left\{t_{l} \leq x \leq t_{u}\right\}=\int_{t_{l}}^{t_{u}} f(x) \mathrm{d} x=1-\alpha,
$$

where $t_{l}$ and $t_{u}$ are the lower and upper bounds of the threshold range, $f(x)$ is the probability density function of each fault multiple feature, and $(1-\alpha)$ is the confidence level.

By comparing the threshold range of fault multiple features with different confidence levels, the optimal confidence level of fault multiple features was determined. The confidence levels of fault multiple features were set to $95 \%$, $90 \%, 85 \%$, and $80 \%$, and the performance of different confidence levels was discussed. As shown in Figure 5, the threshold ranges of fault multiple features with different confidence levels were calculated. As can be seen from Figure 5, the larger the confidence level setting is, the larger the upper bound of the threshold range is and the smaller the lower bound of the threshold range is. It means that, as the confidence level increases, the threshold range becomes larger.

Table 4 shows the overbound rates for different confidence levels which can be used as a reference for the threshold range setting of the fault multiple features. It can be seen from Table 4 that the smaller the confidence level is, the larger the overbound rates' data and overbound rates are. In order to avoid a faulty motor being misjudged as a HM, the confidence level of fault diagnosis for the HM was set to $90 \%$, and the confidence level of the faulty motor was set to $95 \%$ according to the results in Figure 4 and Table 4 . The threshold ranges of the HM with a confidence level of $90 \%$ were calculated by equation (10) as shown in Figure 6.

By modeling the probability distribution of fault indicators in different fault conditions, the threshold ranges of fault multiple features with a confidence level of $90 \%$ were calculated by equation (10). Figure 7 shows the threshold ranges of the ISFM with a confidence level of $95 \%$. The upper and lower bounds of the threshold ranges for each fault multiple feature are shown in Table 5, in which $f_{m}=k c p n / 60$, where $k, c, p$, and $n$ are the number of commutators, the coefficient determined by the number of commutators, the number of pole pairs, and the rotational speed, respectively. The speed range of the HM is $5400-6700 \mathrm{r} / \mathrm{min}$, so the threshold range of $f_{m}$ is $[540,670]$. Similarly, the threshold ranges of $f_{m}$ for other faulty motors are shown in Table 5. It can be seen from Table 5 that the threshold ranges of $P_{D C}$ of the HM and $P_{D C}$ of the OWFM do not overlap, and the HM and the OWFM can be diagnosed.

3.5. Fault Diagnosis Algorithm. The fault classification indicator $d_{i}(m)$ was defined to measure the membership degree of the tested motor $(m)$ belonging to the $i$ th motor type (HM, ISFM, OWFM, WDFM, and AMFM), and it can be calculated by the following equation:

$$
d_{i}(m)=\prod_{j=1}^{j=4} u_{i j}\left(m_{j}\right), \quad(i=1,2,3,4,5 ; j=1,2,3,4),
$$

where $m_{j}$ is the $j$ th fault multiple features of the tested motor $m$. $u_{i j}\left(m_{j}\right)$ reflects whether $m_{j}$ is in the threshold range of the $i$ th motor type, and it can be calculated by the following equation:

$$
u_{i j}\left(m_{j}\right)= \begin{cases}1, & t_{i j}^{l} \leq m_{j} \leq t_{i j}^{u} \\ 0, & m_{j} \leq t_{i j}^{l} \text { or } m_{j} \geq t_{i j}^{u}\end{cases}
$$

where $t_{i j}^{l}$ and $t_{i j}^{u}$ are the lower and upper bound of the threshold range of the $j$ th fault multiple features for the $i$ th motor type (Table 4). According to equation (13), the tested motor was classified. 

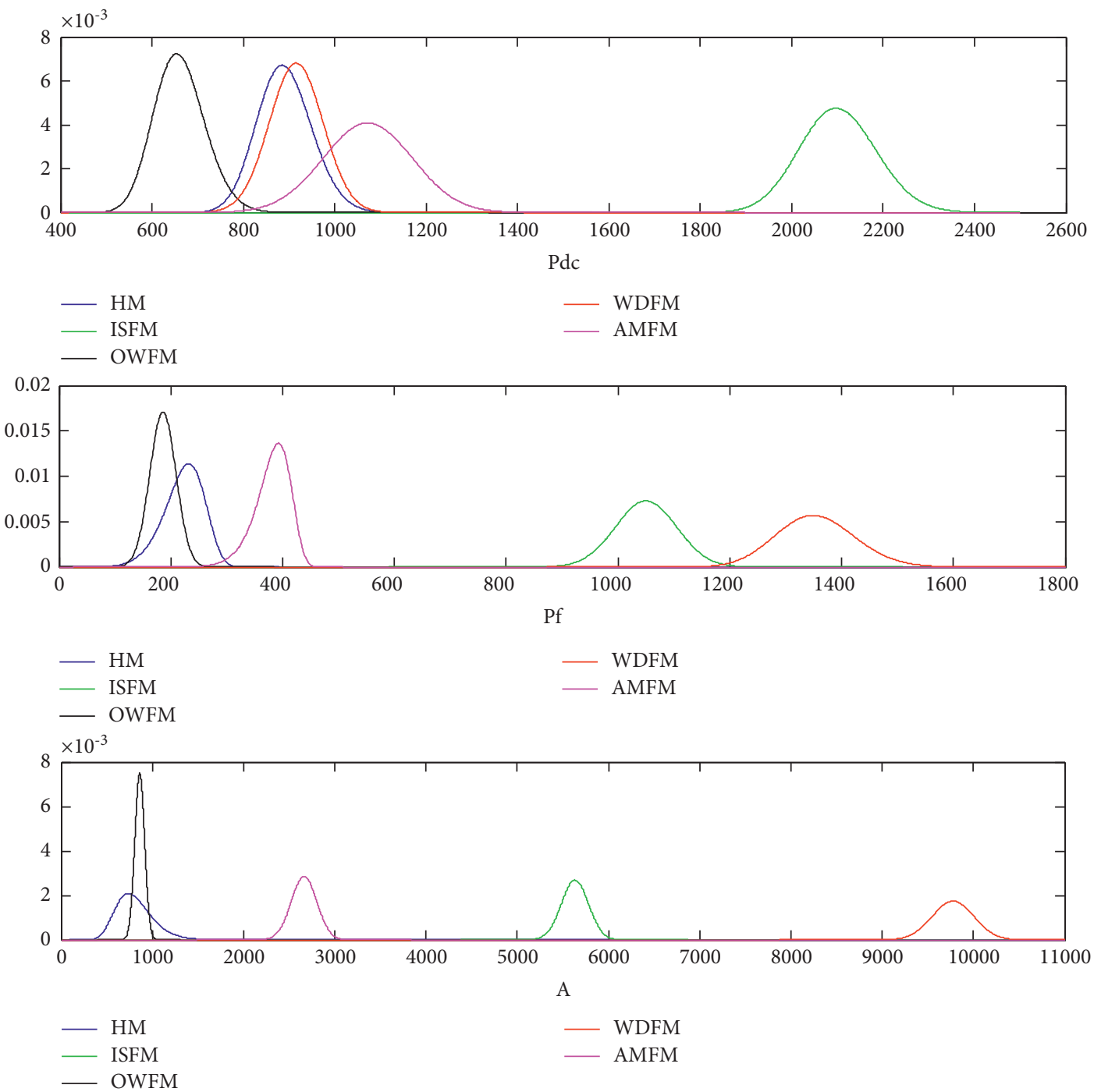

Figure 4: Probability density functions of the fault multiple features in different conditions.

$\left\{\begin{array}{l}\text { If } d_{1}(m)=1, \text { then the tested motor is a } H M, \\ \text { if } d_{2}(m)=1, \text { then the tested motor is a ISFM, } \\ \text { if } d_{3}(m)=1, \text { then the tested motor is an OWFM, } \\ \text { if } d_{4}(m)=1, \text { then the tested motor is a } W D F M, \\ \text { if } d_{5}(m)=1, \text { then the tested motor is a } A M F M .\end{array}\right.$

3.6. Steps of the Fault Diagnosis Method. Based on the above analysis, the MDCM fault diagnosis method is proposed and shown in Figure 8. The steps of the proposed fault diagnosis method can be summarized as follows.

Step 1. Data acquisition.

The armature currents are processed by a preamplifier, a low-pass filter, an A/D converter, and FFT transformation, and the values of fault multiple features are obtained.

Step 2. Confirm the probabilistic model.

Build the probability distribution model of fault multiple features, calculate the overall average membership advantage of each fault multiple feature, and then determine the probabilistic model of each fault multiple feature based on the advantage criterion of the maximum overall average membership.

Step 3. Calculate threshold ranges.

Calculate the threshold ranges of each fault multiple feature using the probability model.

Step 4. Fault diagnosis.

Calculate the fault classification indicator, and then give the fault diagnosis result of the tested motor based on the fault diagnosis algorithm. 


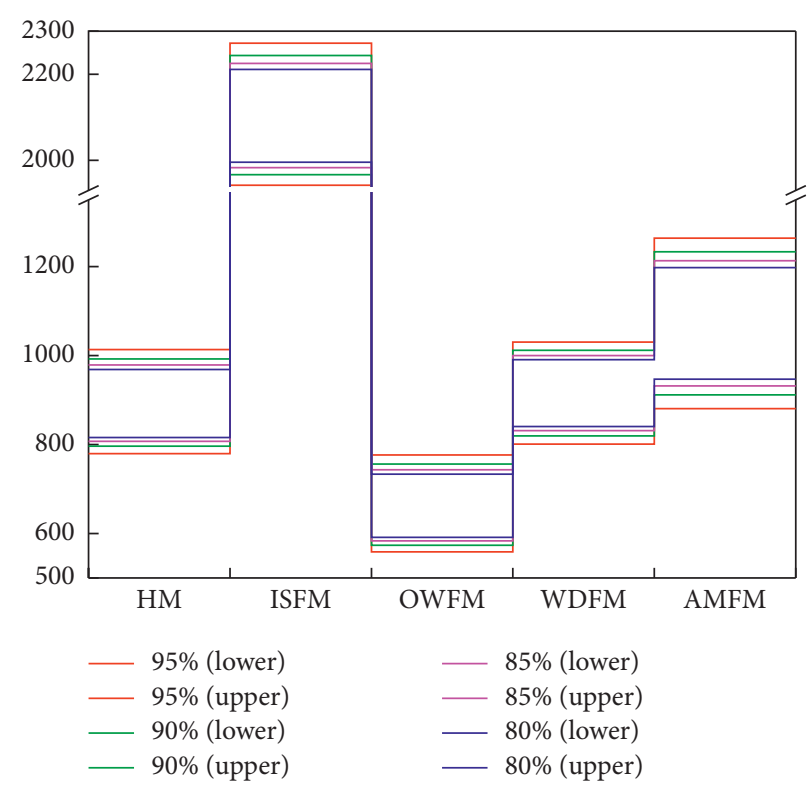

(a)

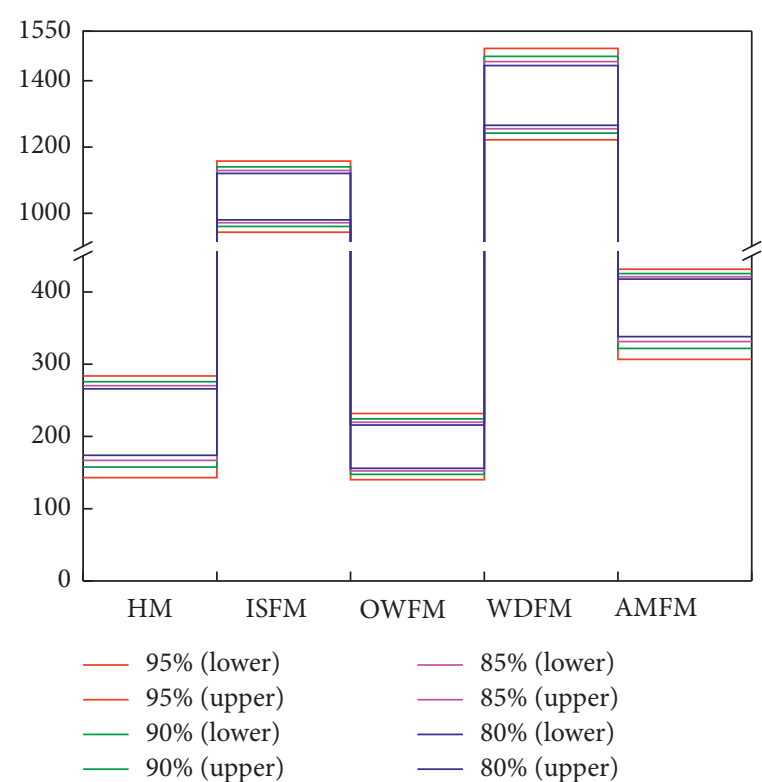

(b)

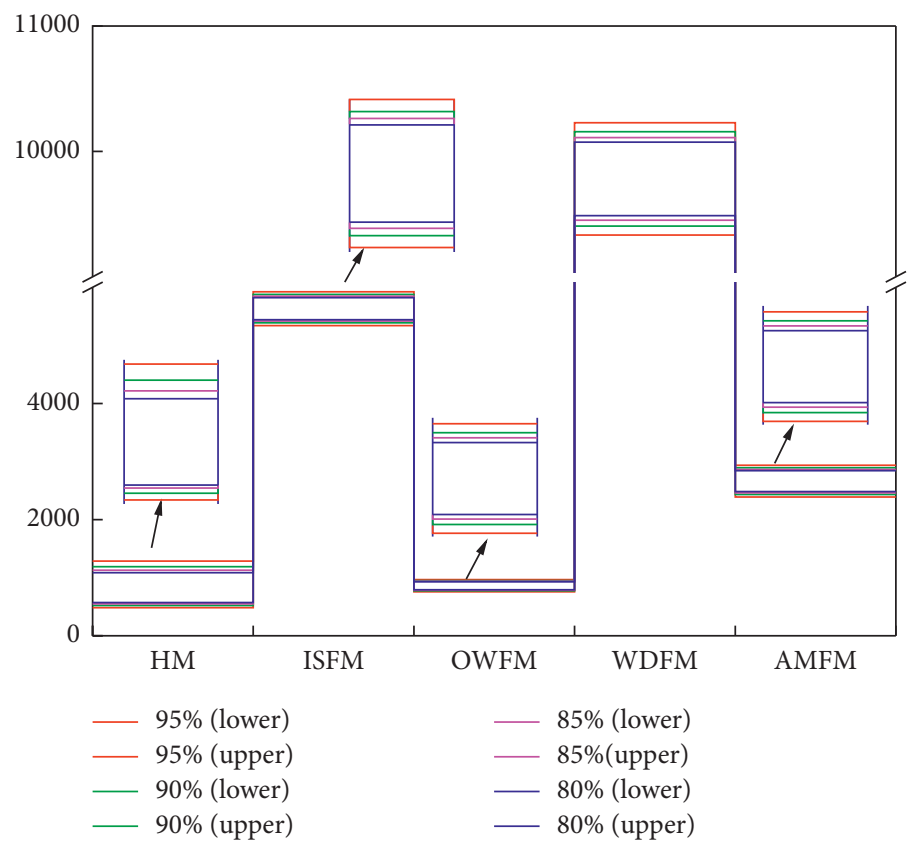

(c)

Figure 5: Threshold range of fault multiple features with different confidence levels: (a) $P_{d c}$, (b) $P_{f}$, and (c) $A$.

\section{Performance Analysis of the Fault Diagnosis Method}

4.1. Diagnostic Device. In order to verify the validity of the fault diagnosis method, the fault diagnosis experiments were performed. The diagnostic device of MDCMs is shown in Figure 9, which mainly includes the data collection and processing module, the microprocessing module, and the LCD display module. The function of the signal acquisition and processing module is to amplify the current signal and remove the high-frequency noise generated by the commutation. The STM32F107VCT chip is selected as the processor of the microprocessor module, which mainly plays the role of A/D sampling, FFT transformation, and fault diagnosis. The LCD display module mainly displays the fault multiple feature values and diagnosis results. 100 healthy motors and 30 motors (Xiamen Dazhen Electric Co., Ltd.) with each fault type were selected to test the proposed method.

4.2. Diagnostic Results. The fault diagnosis results of motors with different faults are shown in Table 6 . Table 6 shows that one of the $100 \mathrm{HMs}$ is misjudged as other faults, and the 
TABLE 4: Overbound rates for different confidence levels.

\begin{tabular}{|c|c|c|c|c|c|c|}
\hline Confidence level (\%) & Fault multiple features & HM (\%) & ISFM (\%) & OWFM (\%) & WDFM (\%) & $\operatorname{AMFM}(\%)$ \\
\hline \multirow{3}{*}{95} & $P_{d c}$ & 2.73 & 5.00 & 3.33 & 6.67 & 3.33 \\
\hline & $P_{f}$ & 2.73 & 6.67 & 5.00 & 3.33 & 1.67 \\
\hline & $A$ & 0.91 & 6.67 & 6.67 & 6.67 & 3.33 \\
\hline \multirow{3}{*}{90} & $P_{d c}$ & 10.91 & 10.00 & 3.33 & 8.33 & 5.00 \\
\hline & $P_{f}$ & 7.27 & 11.67 & 10.00 & 8.33 & 11.67 \\
\hline & $A$ & 8.18 & 8.33 & 10.00 & 10.00 & 6.67 \\
\hline \multirow{3}{*}{85} & $P_{d c}$ & 16.36 & 11.67 & 6.67 & 10.00 & 13.33 \\
\hline & $P_{f}$ & 13.64 & 16.67 & 16.67 & 15.00 & 18.33 \\
\hline & $A$ & 17.27 & 10.00 & 13.33 & 11.67 & 16.67 \\
\hline \multirow{3}{*}{80} & $P_{d c}$ & 20.00 & 15.00 & 10.00 & 13.33 & 25.00 \\
\hline & $P_{f}$ & 24.55 & 21.67 & 23.33 & 20.00 & 26.67 \\
\hline & $A$ & 25.45 & 13.33 & 15.00 & 16.67 & 20.00 \\
\hline
\end{tabular}
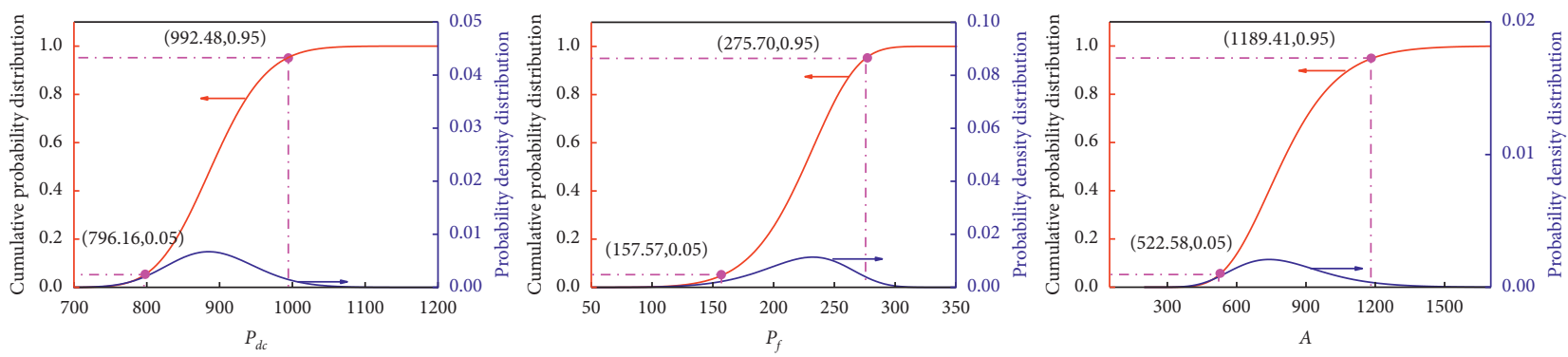

Figure 6: Threshold ranges of fault multiple features for the HM.
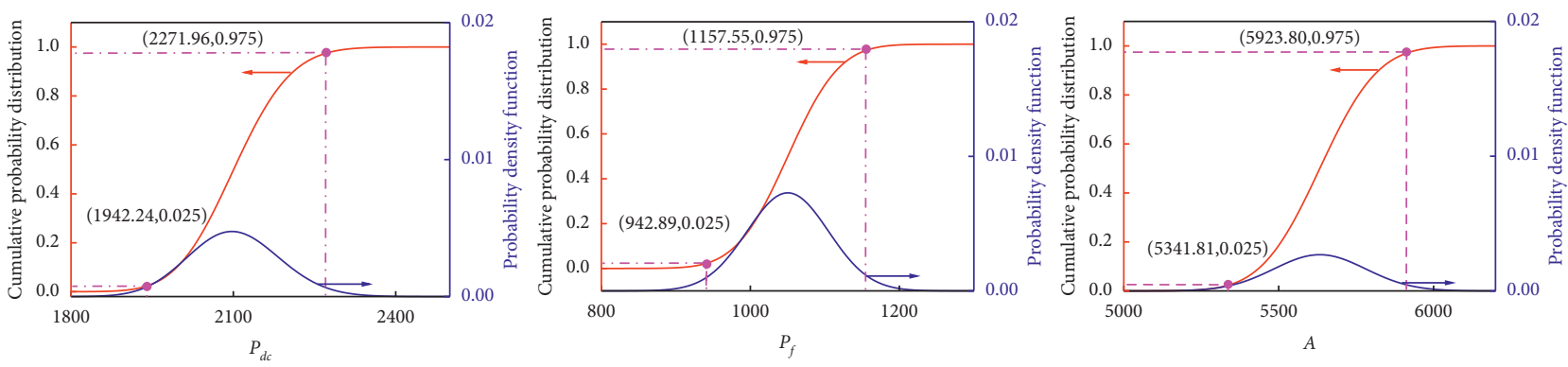

FIGURE 7: Threshold ranges of fault multiple features for the ISFM.

TABLE 5: Threshold of the fault multiple features in different conditions.

\begin{tabular}{lcccccccc}
\hline \multirow{2}{*}{ Type of fault } & \multicolumn{2}{c}{$P_{d c}$} & \multicolumn{2}{c}{$f_{m}$} & \multicolumn{2}{c}{$P_{f}$} & \multicolumn{2}{c}{$A$} \\
& $t_{1}^{l}$ & $t_{1}^{u}$ & $t_{2}^{l}$ & $t_{2}^{u}$ & $t_{3}^{l}$ & $t_{3}^{u}$ & $t_{4}^{l}$ \\
\hline HM & 796.16 & 992.48 & 540.00 & 670.00 & 157.57 & 275.70 & 522.58 \\
ISFM & 1942.24 & 2271.96 & 160.00 & 240.00 & 942.89 & 1157.55 & 5341.81 & 5923.80 \\
OWFM & 558.56 & 776.38 & 560.00 & 600.00 & 140.10 & 231.74 & 757.49 & 965.99 \\
WDFM & 800.86 & 1030.46 & 160.00 & 200.00 & 1221.80 & 1498.05 & 9333.27 & 1022.86 \\
AMFM & 880.73 & 1264.07 & 600.00 & 680.00 & 306.73 & 431.46 & 2388.89 & 2936.90 \\
\hline
\end{tabular}

accuracy rate of $\mathrm{HM}$ is $99 \%$. The reason is that the confidence level of HM was set to $90 \%$, and the threshold ranges of fault multiple features were narrowed accordingly. From Table 6, the accuracy rate of ISFM, OWFM, WDFM, and
AMFM is, respectively, $96.67 \%, 96.67 \%, 100 \%$, and $93.33 \%$, and the faulty motor has not been diagnosed as the healthy motor. The reason is that the confidence levels of ISFM, OWFM, WDFM, and AMFM were all set to $95 \%$. 


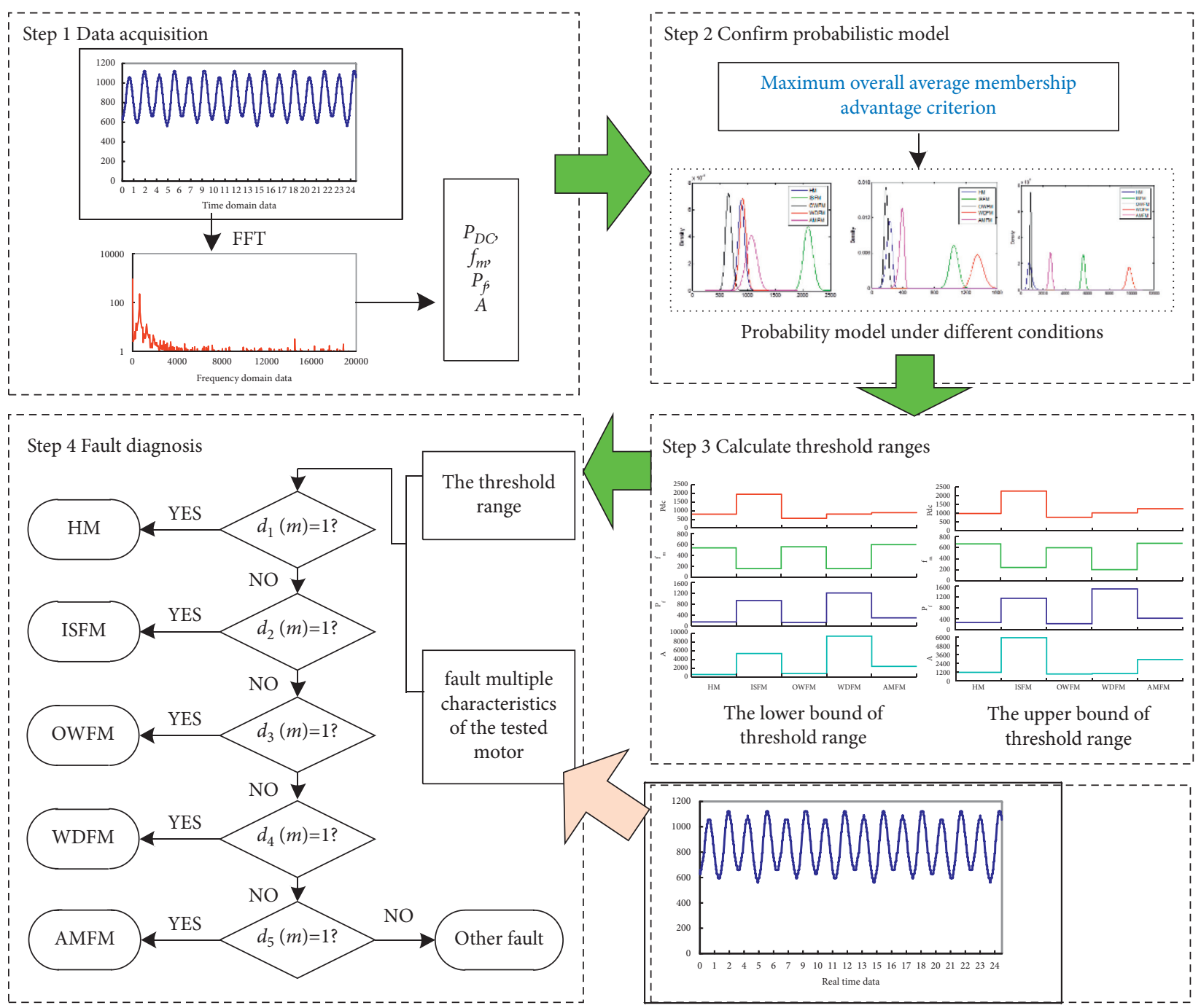

FIgURE 8: The flowchart of fault diagnosis.
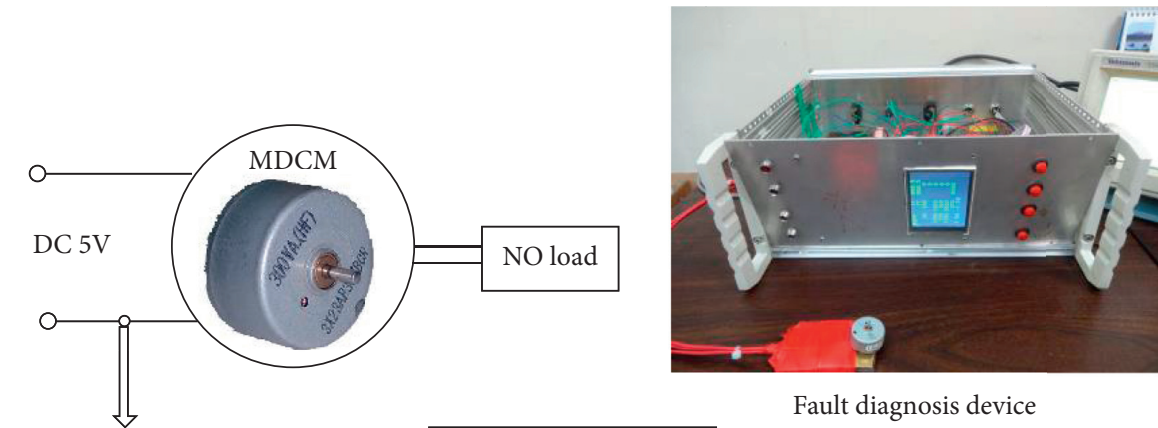

Fault diagnosis device

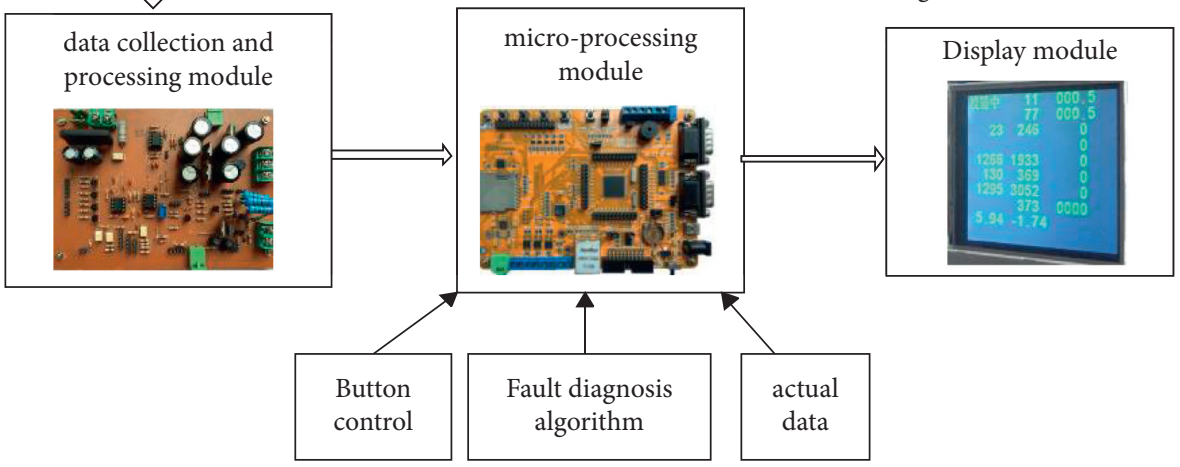

Figure 9: View of the fault diagnosis device. 
Table 6: Diagnostic results of motors with different faults.

\begin{tabular}{|c|c|c|c|c|c|c|c|}
\hline Type of fault & $\mathrm{HM}$ & ISFM & OWFM & WDFM & AMFM & Other faults & Accuracy rate (\%) \\
\hline $\mathrm{HM}$ & 99 & 0 & 0 & 0 & 0 & 1 & 99 \\
\hline ISFM & 0 & 29 & 0 & 0 & 0 & 1 & 96.67 \\
\hline OWFM & 0 & 0 & 29 & 0 & 0 & 1 & 96.67 \\
\hline WDFM & 0 & 0 & 0 & 30 & 0 & 0 & 100 \\
\hline AMFM & 0 & 0 & 0 & 0 & 28 & 2 & 93.33 \\
\hline
\end{tabular}

Table 7: Diagnostic results of motors on the production line.

\begin{tabular}{lcc}
\hline Type of fault & Proposed method & Manual diagnosis \\
\hline HM & 2990 & 2990 \\
Other faults & 8 & 1 OWFM, 7 HMs \\
WDFM & 2 & 2 WDFMs \\
\hline
\end{tabular}

To further calculate the diagnostic accuracy of the proposed method, 3000 motors were randomly selected from the production line, and the diagnostic accuracy of motors is calculated and shown in Table 7. It can be seen from Table 7 that the accuracy rate of HMs is $99.76 \%$, and the faulty motor has not been diagnosed as the healthy motor. It means that the proposed method can be applied to the motor factory testing.

\section{Conclusions}

This paper proposes a probability modeling approach by the threshold ranges of each fault multiple feature. By calculating the overall average membership advantage of the four distribution functions, the probability density of fault multiple features can be determined; then, the threshold ranges of fault multiple features can be obtained. Finally, the validity of the fault diagnosis method is verified. In this paper, the main findings and the shortcomings can be summarized as follows:

(1) The membership functions of the normal distribution, exponential distribution, Weibull distribution, and lognormal distribution are established to calculate the maximum overall average membership advantage of the fault multiple features.

(2) The maximum overall average membership advantage criterion is used to determine the probability distribution model of fault multiple features, which solves the problem that the classical statistical analysis is difficult to determine the optimal probability distribution model.

(3) The threshold ranges of fault multiple features are calculated by setting the confidence of the probability density functions. The motor fault diagnosis algorithm is given based on the threshold range of fault multiple features.

(4) When the proposed method is applied to other types of motors, the probability model needs to be retrained.

\section{Data Availability}

The feature data of motor faults used to support the findings of this study are included within the article.

\section{Conflicts of Interest}

The authors declare no conflicts of interest.

\section{Acknowledgments}

The work described in this paper was supported by the Doctoral Research Project of Guizhou Normal University, Major Science and Technology Projects of Guizhou ([2019] 3003), Natural Science Foundation of Guizhou Province ([2019]1233), Technology Support Programme of Guizhou ([2018]2199), and Special Fund of Chinese Central Government Guidance for Local Science and Technology Development ([2016] 4006).

\section{References}

[1] S.-I. Moriyama, "Torque measurement method based on the lorentz force law for miniature dc motor," IEEJ Transactions on Electrical and Electronic Engineering, vol. 13, no. 2, pp. 322-329, 2018.

[2] Z. Xie, W. Wu, and J. Hong, "Study on properties of armature current for micro DC motors and multiple features of fault diagnosis," Electric Machines and Control, vol. 19, no. 8, pp. 107-114, 2015.

[3] A. Bellini, F. Filippetti, C. Tassoni, and G.-A. Capolino, "Advances in diagnostic techniques for induction machines," IEEE Transactions on Industrial Electronics, vol. 55, no. 12, pp. 4109-4126, 2008.

[4] H. Henao, G.-A. Capolino, M. Fernandez-Cabanas, F. Filippetti, C. Bruzzese, and E. Strangas, "Trends in fault diagnosis for electrical machines a review of diagnostic techniques," Ieee Industrial Electronics Magazine, vol. 8, no. 2, pp. 31-42, 2014.

[5] M. Heydarzadeh, M. Zafarani, M. Nourani, and B. Akin, “A wavelet-based fault diagnosis approach for permanent magnet synchronous motors," IEEE Transactions on Energy Conversion, vol. 34, no. 2, pp. 761-772, 2019.

[6] B. R Nayana and P. Geethanjali, "Analysis of statistical timedomain features effectiveness in identification of bearing faults from vibration signal," IEEE Sensors Journal, vol. 17, no. 17, pp. 5618-5625, 2017.

[7] A. Glowacz and Z. Glowacz, "Recognition of rotor damages in a dc motor using acoustic signals," Bulletin of the Polish Academy of Sciences, Technical Sciences, vol. 65, no. 2, pp. 187-194, 2017.

[8] G. Singh, T. C. Kumar, and V. N. Naikan, "Induction motor inter turn fault detection using infrared thermographic 
analysis," Infrared Physics \& Technology, vol. 77, pp. 277-282, 2016.

[9] M. Seera, C. P. Lim, D. Ishak, and H. Singh, "Fault detection and diagnosis of induction motors using motor current signature analysis and a hybrid FMM-CART model," IEEE Transactions on Neural Networks, vol. 23, no. 1, pp. 97-108, 2012.

[10] G. Joksimovic and J. Penman, "The detection of inter-turn short circuits in the stator windings of operating motors," IEEE Transactions on Industrial Electronics, vol. 47, no. 5, pp. 1078-1084, 2000.

[11] S. Moon, H. Jeong, H. Lee, and S. W. Kim, "Interturn short fault diagnosis in a pmsm by voltage and current residual analysis with the faulty winding model," IEEE Transactions on Energy Conversion, vol. 33, no. 1, pp. 190-198, 2018.

[12] O. Moseler and R. Isermann, "Application of model-based fault detection to a brushless dc motor," IEEE Transactions on Industrial Electronics, vol. 47, no. 5, pp. 1015-1020, 2000.

[13] C. Chan, S. Hua, and H. Zhang, "Application of fully decoupled parity equation in fault detection and identification of dc motors," IEEE Transactions on Industrial Electronics, vol. 5 , no. 4 , pp. $1277-1284,2006$.

[14] H. M. Usman, S. Mukhopadhyay, and H. Rehman, "Permanent magnet $\mathrm{dc}$ motor parameters estimation via universal adaptive stabilization," Control Engineering Practice, vol. 90, pp. 50-62, 2019.

[15] S. Munikoti S, L. Das, B. Natarajan, and B. Srinivasan, "Datadriven approaches for diagnosis of incipient faults in $\mathrm{dc}$ motors," Ieee Transactions on Industrial Informatics, vol. 15, no. 9, pp. 5299-5308, 2019.

[16] S. Lu, G. Qian, Q. He, F. Liu, Y. Liu, and Q. Wang, "In situ motor fault diagnosis using enhanced convolutional neural network in an embedded system," IEEE Sensors Journal, vol. 20, no. 15, pp. 8287-8296, 2020.

[17] X. Xu, X. Qiao, N. Zhang, J. Feng, and X. Wang, "Review of intelligent fault diagnosis for permanent magnet synchronous motors in electric vehicles," Advances in Mechanical Engineering, vol. 12, no. 7, Article ID 1687814020944323, 2020.

[18] J. Burriel-Valencia, R. Puche-Panadero, J. Martinez-Roman et al., "Automatic fault diagnostic system for induction motors under transient regime optimized with expert systems," Electronics, vol. 8, no. 1, p. 6, 2019.

[19] X. Li, W. Zhang, Q. Ding, and X. Li, "Diagnosing rotating machines with weakly supervised data using deep transfer learning," Ieee Transactions on Industrial Informatics, vol. 16, no. 3, pp. 1688-1697, 2020.

[20] Z. Guo, M. Liu, H. Qin, and B. Li, "Mechanical fault diagnosis of a dc motor utilizing united variational mode decomposition, sampen, and random forest-sprint algorithm classifiers," Entropy, vol. 21, no. 5, p. 470, 2019.

[21] W. Sun, R. Zhao, R. Yan, S. Shao, and X. Chen, "Convolutional discriminative feature learning for induction motor fault diagnosis," Ieee Transactions on Industrial Informatics, vol. 13, no. 3, pp. 1350-1359, 2017.

[22] D. Xiao, Y. Huang, L. Zhao, C. Qin, H. Shi, and C. Liu, "Domain adaptive motor fault diagnosis using deep transfer learning," IEEE Access, vol. 7, pp. 80937-80949, 2019.

[23] H. Wang, J. Zhao, Q. Sun, and H. Zhu, "Probability modeling for pv array output interval and its application in fault diagnosis," Energy, vol. 189, Article ID 116248, 2019.

[24] G. An and H. Li, "Degradation state identification of cracked ultrasonic motor by means of fault feature extraction method," Shock and Vibration, vol. 2019, Article ID 5180590, 13 pages, 2019.
[25] A. Jafari, J. Faiz, and M. A. Jarrahi, "A simple and efficient current-based method for interturn fault detection in BLDC motors," Ieee Transactions on Industrial Informatics, vol. 17, no. 4, pp. 2707-2715, 2021.

[26] M. Z. Ali and X. Liang, "Threshold-based induction motors single- and multifaults diagnosis using discrete wavelet transform and measured stator current signal," Canadian Journal of Electrical and Computer Engineering-Revue Canadienne De Genie Electrique Et Informatique, vol. 43, no. 3, pp. 136-145, 2020.

[27] M. Irfan, N. Saad, R. Ibrahim, V. Sagayan Asirvadam, and M. Magzoub, "An online fault diagnosis system for induction motors via instantaneous power analysis," Tribology Transactions, vol. 60, no. 4, pp. 592-604, 2017.

[28] H. Liu, Y. Wang, Y. Yang, R. Liao, Y. Geng, and L. Zhou, “A failure probability calculation method for power equipment based on multi-characteristic parameters," Energies, vol. 10, no. 5, p. 704, 2017.

[29] Y. Li, W. Dai, T. Huang, M. Shi, and W. Zhang, "Adaptive early warning method based on similar proportion and probability model," Applied Sciences-Basel, vol. 10, no. 12, p. $4278,2020$.

[30] J. Wen and H. Gao, "Degradation assessment for the ball screw with variational autoencoder and kernel density estimation," Advances in Mechanical Engineering, vol. 10, no. 9, Article ID 1687814018797261, 2018.

[31] Z. Xie, J. Zheng, and Y. Lang, "Modelling and simulation of interturn short fault in micro direct-current motors," International Journal of Power and Energy Systems, vol. 41, no. 2, pp. 122-129, 2021.

[32] J. Xie and C. Liu, Method and Application of Fuzzy Mathematics, Huazhong University of Science and Technology Press, Wuhan, China, 3rd edition, 2006.

[33] H. Zhang and Z. He, Principles and Applications of Fuzzy Diagnosis, Xi'an Jiaotong University Press, Xi'an, China, 1992. 